\title{
Strong piezoelectric effects in unstrained GaN quantum wells
}

\author{
R. Langer *, J. Simon*, O. Konovalov*, N.T. Pelekanos*, R. André**, A. Barski*, \\ M. Godlewski*** \\ *Département de Recherche Fondamentale sur la Matière Condensée, CEA/Grenoble, \\ SP2M/PSC, 38054 Grenoble, Cedex 9, France \\ ** Laboratoire de Spectrométrie Physique, CNRS, Université J. Fourier, 38402 Saint \\ Martin d'Hères, France \\ *** Institute of Physics, Polish Academy of Sciences, 02-668 Warsaw, Al. Lotnikow 32/46 \\ Poland
}

\section{Abstract}

Optical properties of GaN/AlGaN single quantum well (SQW) and multi quantum well (MQW) structures grown by nitrogen plasma assisted MBE on MOCVD-grown GaN/sapphire have been characterised by low temperature photoluminescence. Photoluminescence (PL) peaks corresponding to emission from very narrow (10 $\AA$ wide) SQWs are blue shifted with respect to the bulk GaN emissions but reveal strong red shift for wider SQWs (40 and $60 \AA$ wide). Structural properties of SQW and MQW structures have been characterised by X-ray reciprocal lattice mapping in order to determine the strain conditions and composition of ternary AlGaN alloys. The results clearly demonstrate that in all structures under investigation the ternary (barrier) alloys are fully strained to underlying MOCVDgrown GaN/sapphire substrates. Despite of the fact that GaN quantum wells $(\mathrm{QW})$ are unstrained, photoluminescence experiments clearly reveal the presence of extremely strong electric fields in the QW regions, which can be attributed to the interplay of the piezoelectric-type polarisation in the well and barrier layers due to Fermi level alignment. 


\section{Introduction}

GaN/AlGaN QWs are subject of intense investigations due to their potential for electronic and opto-electronic applications. Many recent publications report on the properties of GaN/AlN and GaN/AlGaN heterostructures, superlattices and/or multi QW structures [1,2,3,4]. Recently, a strong piezoelectric field has been found in GaN/AlGaN heterostructures with strained QW regions which was attributed to strain induced phenomena [1]. We report here the results on GaN/AlGaN SQW and MQW structures grown by Radio Frequency Molecular Beam Epitaxy (RFMBE) on MOCVD-grown GaN/sapphire. In order to demonstrate that the strong electric field is present in unstrained quantum wells, the total thickness of AlGaN barriers in all our samples was kept below the critical thickness and hence the $\mathrm{AlGaN}$ layers are strained. Our structures are pseudomorphic with the GaN/sapphire substrate and the GaN QW's are strain free.

\section{Experimental}

The GaN/AlGaN SQW structures studied have a single GaN QW of 10 and $40 \AA$ thickness embedded between $1000 \AA$ thick AlGaN barriers. The Al fraction in the barriers was about 17$20 \%$, as determined independently from PL and X-rays experiments. MQW structures of 20, 40 and $60 \AA$ thick GaN wells have been grown for two different Aluminium concentrations in GaAlN barriers respectively of 10 and $20 \%$. The sample growth was performed in a RIBER MBE 2300 chamber. Standard Knudsen cells were used for Gallium and Aluminium evaporation and the active nitrogen was generated by a commercial (ADDON) radio frequency (RF) cell. Growth was performed at a relatively low substrate temperature of $650^{\circ} \mathrm{C}$. The composition of the GaAlN films was adjusted by varying the beam equivalent pressure of the Gallium and Aluminium fluxes. The thickness of the layers was controlled by RHEED oscillations. MBE growth was performed on $1.5 \mu \mathrm{m}$ thick GaN layers grown by MOCVD on sapphire. The oxide on this MOCVD grown GaN surface can easily be removed by acid etching (regal water) and short heating $\left(\sim 650^{\circ} \mathrm{C}\right)$ prior to growth. A well-defined RHEED pattern and intense RHEED oscillations show that the treated MOCVD surface is smooth on an atomic scale. To completely get rid of the eventual influence from a surface oxide or from a contamination layer, we interpose a few 1000 Åthick GaN layer.

PL time resolved measurements were performed at $2 \mathrm{~K}$ with pulsed excitation (of ns length) above QW band gap energy and photon counting system with multi-channel analyser for PL detection. 


\section{Results and discussion}

Low temperature (2 K) PL spectra, shown in Figure 1, are dominated by $3.47 \mathrm{eV}$ band gap emission of the underlying 1.5 $\mu \mathrm{m}$ thick MOCVD-grown epilayer and of MBE-grown (few times 1000 A thick) buffer layer. PL emission of ternary AlGaN barrier layers is also seen on the highenergy side of the emission spectra. In addition to barrier and substrate emissions, a SQW emission is also resolved with its LO-phonon replica. Assuming QW confinement effects only, SQW PL should show a blue-shift with respect to the GaN substrate emission, which should increase with a decreasing QW width. However, as shown in Figure 1, only the PL of $10 \AA$ wide SQW is blue-shifted by about $150 \mathrm{meV}$. For $40 \AA$ A thick SQW's a pronounced red-shift of the PL lines is observed from the expected PL position. This red-shift of the PL can be explained by a strong internal electric field present in the structure [5], as will be discussed later. In order to determine the influence of Aluminium concentration in barriers on the PL line positions, two samples with different Aluminium concentrations have been grown. The first sample consisted of 20, 40 and $60 \AA$ thick quantum wells separated by $100 \AA$ GaAlN barriers with the Aluminium concentration of $10 \%$. The second sample has the same structure but the Aluminium concentration was increased to $20 \%$. Low temperature PL spectra of our MQW structures are shown in Figure 2. Only the emission from 20 A thick well in the low Aluminium content sample is blue-shifted with respect to the GaN substrate emission. All emissions from 40 and 60 Åthick wells are detected below the GaN emission. This phenomenon is clear evidence for a strong electric field present in our structures, which increases with the Aluminium concentration in barriers.

In order to determine the origin of this field the strain present in our samples has to be investigated. Strain could be precisely determined by X-rays reciprocal lattice mapping. Results of such experiments are shown in Figure 3 for the $40 \AA$ thick SQW. X-rays mapping around the (-2 0 2) Bragg peak of the GaN substrate is shown. These measurements clearly demonstrate that the $\mathrm{AlGaN}$ alloy has the same in-plane lattice parameter (represented by the (h, $\mathrm{k}$ ) axis in reciprocal units of $\mathrm{Al}_{2} \mathrm{O}_{3}$ ) as the GaN substrate. Both $1000 \AA$ thick GaAlN barriers are thus fully strained to GaN. Similar conclusions can be drawn from reciprocal space mappings for all other QW's structures under investigation.

For totally pseudomorphic samples, one can precisely determine the Aluminium fraction in GaAlN barriers. From the in-plane lattice parameter and from the lattice parameter along the growth axis, using Vegard's law for the lattice parameter and a linear interpolation between Poisson ratios for GaN [6] and AlN [7], we determine the Al fraction in all our samples, which agrees with our estimations from PL studies. 
Despite of the fact that GaN QW's are not strained in our samples, we observed a dramatic dependence of QW PL on a QW width and on Aluminium concentration in barriers. This can be related to strong piezoelectric effects introducing strong internal electric fields to the structure. This hypothesis is further confirmed by PL kinetics measurements. For 40 and $60 \AA$ wide SQW the PL intensity decay is highly non exponential and in $\mu$ s range. In fact such slow PL kinetics resembles that of the yellow emission band and dramatically varies from the PL kinetics of excitons from GaN substrate. Those excitons show PL decay times in the range of $100 \mathrm{ps.} \mathrm{Slow}$ PL kinetics in GaN/AlGaN QW system can be explained by a strong reduction of radiative recombination probability caused by electric field induced spatial separation of electrons and holes in a wide QW. Strong piezoelectric effects also explain why SQW emission is relatively weak when compared to the substrate emission. As was mentioned above, the internal electric field strongly reduces radiative recombination rates of QW's.

In conclusion, the present studies demonstrate strong piezoelectric effects in GaN/AlGaN QW structures with strained AlGaN barrier layers but with strain free GaN QW's. We believe that this is related to the large piezoelectric constants predicted for GaN and GaAlN [8].

\section{References}

[1] J. S. Im, H. Kollmer, J. Off, A. Sohmer, F. Scholz, A. Hangleiter, Phys. Rev. B 57, R9435 (1998).

[2] D. Korakakis, K. F. Ludwig, T. D. Moustakas, Appl. Phys. Lett. 72, 1004 (1998).

[3] K. C. Zeng, J. Y. Lin, H. X. Jiang, A. Salvador, G. Popovici, H. Tang, W. Kim, H. Morkoc, Appl. Phys. Lett. 71, 1368 (1997).

[4] M. A. L. Johnson, Shizuo Fujita, W. H. Rowland, K. A. Bowers, W. C. Hughes, Y. W. He, N. A. El-Masry, J.W. Cook, J. F. Schetzina, J. Ren, J. A. Edmond, J. Vac. Sci. Technol. B 14, 2349-2353 (1996).

[5] R. Langer, J. Simon, O. Konovalov, N.T. Pelekanos, A. Barski, M. Leszczynski, MRS Internet J. Nitride Semicond. Res. 3, 46 (1998).

[6] C. Kisielowski, J. Krüger, S. Ruvimov, T. Suski, J. W. Ager, E. Jones, Z. Lilienthal-Weber, M. Rubin, M. D. Bremser, R. F. Davis, Phys. Rev. B 54, 17745 (1996).

[7] L.E. McNeil, M. Grimsditch, R.H. French, J. Am. Ceram. Soc. 76, 1132-1136 (1993).

[8] F. Bernardini, V. Fiorentini, D. Vanderbilt, Phys. Rev. B 56, R10024 (1997). 


\section{Figures}

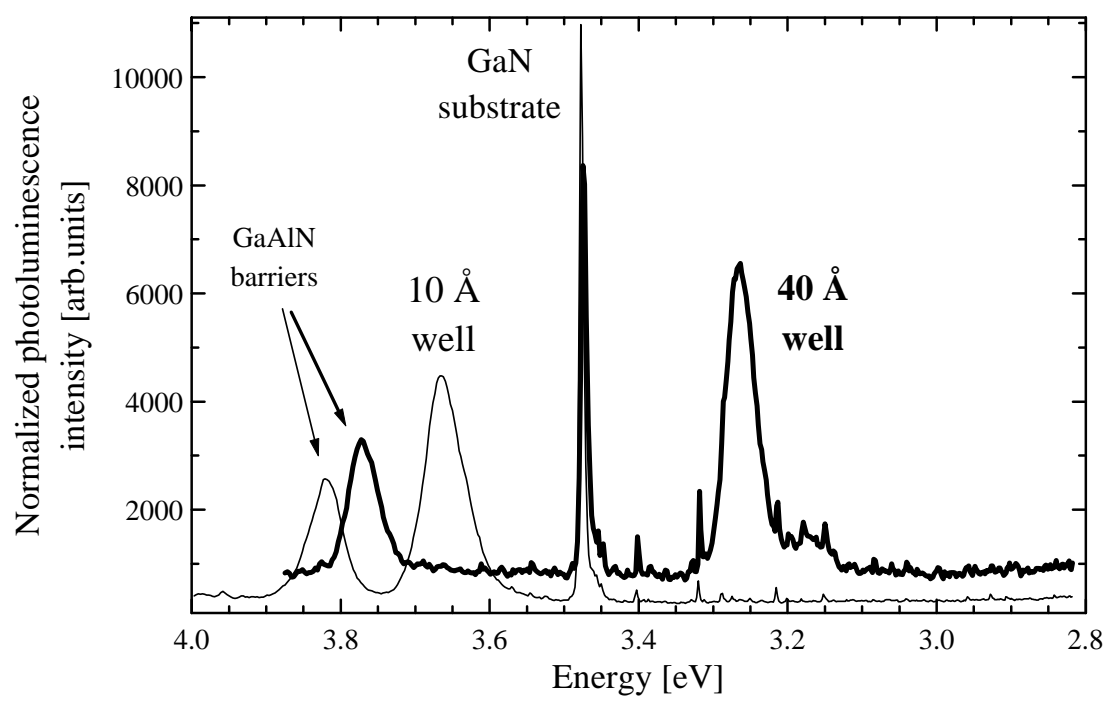

Fig1: Low temperature photoluminescence of $10 \AA$ and $40 \AA$ thick GaN quantum wells in AlGaN barriers with an aluminium concentration of $17.2 \%$ and $20.6 \%$ respectively.

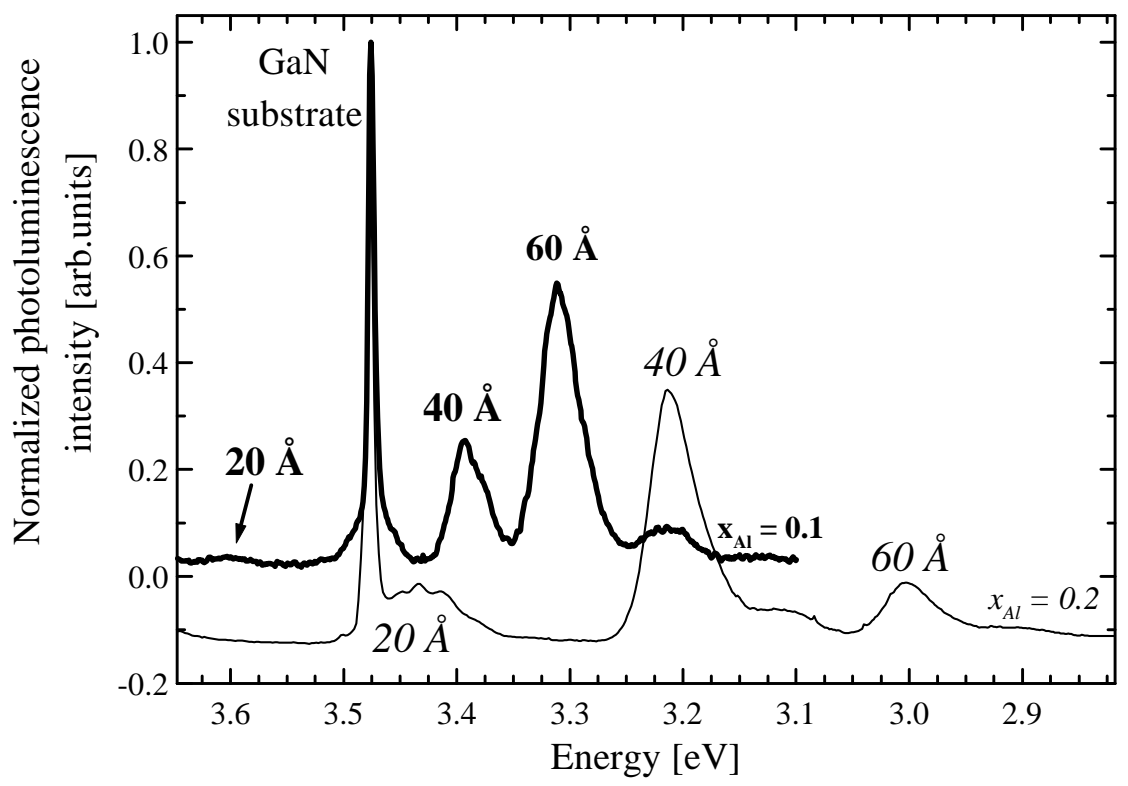

Fig2: Low temperature photoluminescence of two samples with $20 \AA 40 \AA$ and $60 \AA$ thick GaN quantum wells and $100 \AA$ A thick AlGaN barriers with an aluminium concentration of $17.2 \%$ and $20.6 \%$ respectively. 
(a)

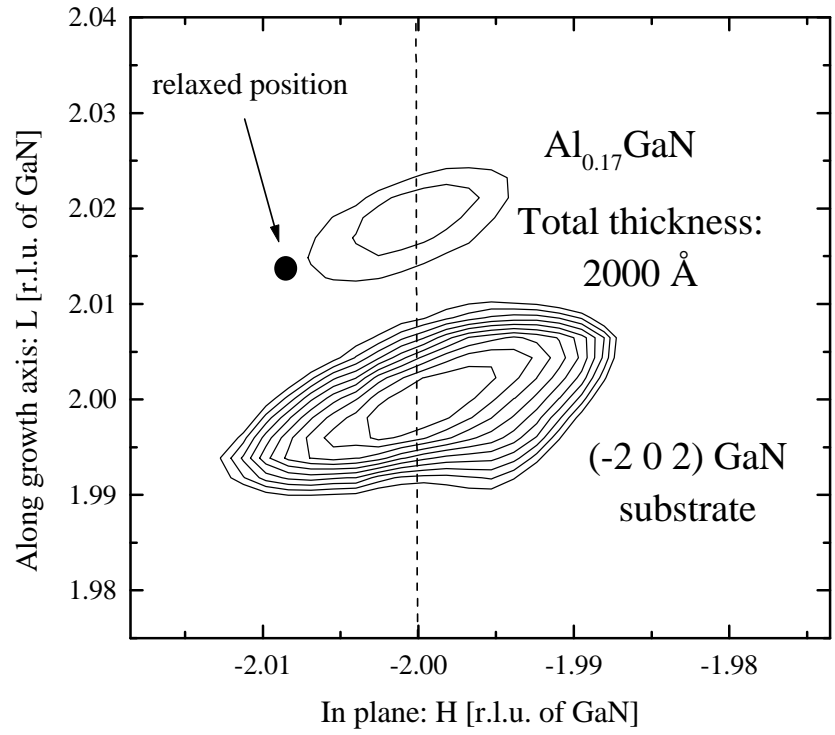

(b)

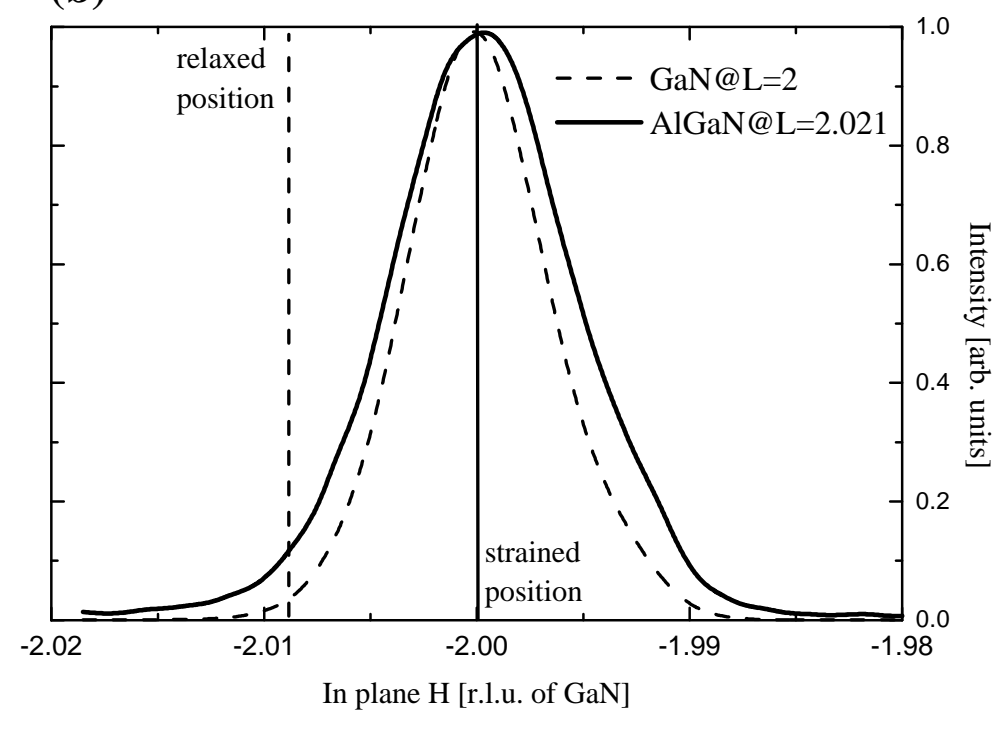

Fig3 (a): X-ray cartography of GaN single quantum well 40 A thick embedded in $1000 \AA$ thick AlGaN barriers around (-2 0 2) Bragg peak of GaN substrate. The AlGaN barriers have the same in-plane lattice parameter than the $\mathrm{GaN}$ substrate. This is shown very precisely on the in plane scans (b) where the in-plane lattice parameter is represented by the $\mathrm{H}$-axis in reciprocal lattice units of $\mathrm{GaN}$ 